\title{
Actualización de las estimaciones de la recarga en el sector central del Valle Argentino, provincia de La Pampa, Argentina
}

\author{
Eduardo E. Mariño(1), Carlos J. Schulz ${ }^{(1)}$, Natalia Loyola ${ }^{(1)}$ y Eduardo C. Castro ${ }^{(1,2)}$ \\ (1) Universidad Nacional de La Pampa- Facultad de Ciencias Exactas y Naturales. \\ Ruta Nac. №35 km 334, (6300) Santa Rosa, La Pampa. \\ emarinio@exactas.unlpam.edu.ar \\ (2) Universidad Nacional de La Pampa- Facultad de Ciencias Humanas. Cnel. Gil 353, (6300) Santa Rosa, La Pampa.
}

\begin{abstract}
RESUMEN
Los procesos que gobiernan la recarga presentan dificultades para su cuantificación debido a la variabilidad espacio-temporal de las condiciones hidroclimáticas y las características particulares de los sistemas acuíferos. Así, los métodos existentes proporcionan, en ocasiones, estimaciones con un alto índice de incertidumbre. El objetivo del trabajo ha sido la estimación de la recarga en el sector central del Valle Argentino, cuyo potencial hidrogeológico se asocia a un cordón medanoso que actúa como área de recarga. Se consideró de interés actualizar las estimaciones previas en el área en base a datos de las estaciones General Acha y Resguardo Padre Buodo. Se llevó a cabo un balance hídrico diario, mediante el código Visual Balan y el análisis cuantitativo de fluctuaciones del nivel freático. Los resultados medios anuales obtenidos oscilan entre 35 y 53 $\mathrm{mm}$, con máximos entre 76 y $112 \mathrm{~mm}$ y mínimos entre 0 y $21 \mathrm{~mm}$. Los valores mayores produjeron ascensos de 0,60 a poco más de $1 \mathrm{~m}$, mientras que cuando la recarga es exigua o nula se asocia a descensos entre $0,4 \mathrm{y}$ $0,8 \mathrm{~m}$, debidos al predominio de la descarga subterránea. Ambas fluctuaciones implican una variación significativa del almacenamiento subterráneo del acuífero, el cual es la principal fuente de abastecimiento de varias localidades y actividades productivas de la zona. La integración de estos resultados en un modelo conceptual que explica de la dinámica del sistema hídrico, representa un aporte relevante para la gestión del recurso.
\end{abstract}

Palabras clave: Hidrogeología, recarga, balance hídrico, fluctuaciones freatimétricas.

\section{Improved estimate of groundwater recharge in the central part of Valle Argentino, province of La Pampa, Argentina}

\begin{abstract}
The processes that control the recharge have difficulties for their quantification due to hydroclimatic variability and different particularities of the aquifer system. Sometimes, the different methods for recharge assessment provide results with a high degree of uncertainty. The purpose of this paper is to estimate groundwater recharge in the central part of Valle Argentino, whose hydrogeological potential comes from a sand dune system that plays an important role as recharge area. It was considered interesting to update the previous estimates with new data from the General Acha and Padre Buodo sites. The recharge was obtained from a daily water balance (Visual Balan code) and the water-table fluctuation (WTF) method. The mean yearly values of recharge obtained range from 35 to $53 \mathrm{~mm}$, with maximums between 76 and $112 \mathrm{~mm}$ and minimums between 0 and $21 \mathrm{~mm}$. The greater values caused water table rises from 0.60 to just over $1 \mathrm{~m}$, while a low or null recharge is linked to a drop in water levels of between 0.4 and $0.8 \mathrm{~m}$, caused by the predominance of the groundwater outflow. This fluctuation of water table has a severe impact in the water storage in the aquifer, which represents the main source of groundwater for human and livestock consumption in this area. The integration of results in a conceptual model that explain the hydrodynamic behaviour of the system constitutes a relevant contribution to groundwater resource management.
\end{abstract}

Keywords: hydrogeology, recharge, water balance, water table fluctuations. 


\section{Introducción}

Los acuíferos alojados en ambiente medanoso son los principales recursos hídricos con que cuenta la provincia de La Pampa para el abastecimiento de agua destinada al uso urbano y rural. Las acumulaciones arenosas crean condiciones favorables para la infiltración del agua de lluvia, fuertemente condicionada por el marco climático; sus propiedades hidráulicas permiten una explotación con rendimientos superiores a los obtenidos en otros ámbitos y además incrementan localmente la posibilidad de acceder a agua de mayor aptitud para distintos usos. Estas cualidades dan lugar a su selección como sitios preferenciales para la ubicación de obras de captación. Sin embargo, dichos sistemas son sensibles al régimen de explotación y, cuando éste es inadecuado, se puede producir un marcado deterioro cuantitativo y cualitativo del recurso.

El Valle Argentino, en toda su extensión, provee de agua potable a General Acha y otras localidades menores, satisfaciendo la demanda de casi 29.000 habitantes. Además, abastece el consumo ganadero en los establecimientos rurales de la zona.

Dada la importancia social y productiva de este sistema hídrico para la provincia de La Pampa, resulta comprensible que se hayan realizado numerosos estudios hidrogeológicos. Entre otros, cabe mencionar los trabajos de Cavalié (1985), Castro y Tullio (1990), Universidad Ben Gurion (1998), Dornes y Schulz (2001), Schulz et al. (1997, 1998, 1999 y 2002), Mariño et al. (2002), Schulz (2004), Mariño y Schulz (2008), UNLPam (2015) y Galea (2017).

Algunos de los antecedentes citados formulan consideraciones sobre la recarga del acuífero. EI estudio hidrogeológico de la Hoja General Acha (Cavalié, 1985) asume una recarga media anual de $50 \mathrm{~mm}$. La Universidad Ben Gurion (1998) elaboró un informe sobre las posibilidades de realizar riego y otros usos en el sector comprendido entre Padre Buodo y Chacharramendi, donde estima que la recarga anual oscila entre 22 y $64 \mathrm{~mm} / a$ ño. Otro aporte relevante fue realizado por Schulz (2004), quien estimó la recarga por diferentes métodos y obtuvo un rango amplio de resultados (entre 0 y $57 \mathrm{~mm} /$ año).

El presente trabajo tiene como objetivo actualizar la estimación de la recarga en el sector central del valle Argentino, entre la ciudad de General Acha y el paraje Resguardo Padre Buodo.

Se pretende un abordaje metodológico replicable en otros sistemas hídricos que resultan frágiles por su contexto hidroclimático $y$, en consecuencia, son susceptibles de sufrir un deterioro cuantitativo y/o cualitativo, debido a una potencial sobrexplotación. Por ello, la cuantificación confiable de la recarga constituye un insumo fundamental para la planificación y gestión de los recursos hídricos subterráneos.

\section{Caracterización del área de estudio}

El valle Argentino se sitúa en la región central de la provincia de La Pampa, en una faja transicional entre la región subhúmeda y semiárida (Figura 1). Las precipitaciones registradas en General Acha alcanzan una media anual de $542,8 \mathrm{~mm}$, para el período 1898 2016. La evapotranspiración potencial anual calculada por el método de Thornthwaite, para la localidad mencionada varía de 786 a $837 \mathrm{~mm}$, según el período considerado (Galea, 2017).

El valle es una unidad geomorfológica que se extiende en la provincia de La Pampa, desde aproximadamente Chacharramendi en el Oeste, internándose en la provincia de Buenos Aires hacia el Este. Se trata de una depresión de un ancho de entre 3 y $18 \mathrm{~km}$, que se incrementa hacia el este. Su extensión longitudinal, con una dirección aproximada ENE-OSO, alcanza unos $210 \mathrm{~km}$, presentando un desnivel topográfico que llega a superar los 50 metros con respecto a la planicie que lo limita. Su característica morfológica principal es la presencia de un cordón medanoso central que lo divide, conformando lateralmente dos sectores deprimidos, conocidos localmente como los valles de General Acha, al Sur, y de Utracán, al Norte. Ambos valles están parcialmente ocupados por un conjunto de cuerpos de agua superficial, que se presentan como lagunas o salitrales, dependiendo del estadio del ciclo hidrológico (Schulz, et al., 1999).

Para definir algunos de los parámetros que se utilizarán en el balance hidrológico, es relevante conocer las características principales de los suelos y la vegetación de la región. En tal sentido, los suelos del valle Argentino se pueden enmarcar en una asociación bien manifestada de tres sistemas de suelos que no presentan organización edafogenética (Martínez y Fiorucci, 1998): Ustipsammentes típicos, Calciustoles típicos y Haplustoles ácuicos. La vegetación es predominantemente natural, clasificándosela como un pastizal samófilo, de altura intermedia, perennifolio, denso y con dos estratos definidos, uno de gramíneas intermedias y otro de gramíneas bajas y herbáceas (Cano et al, 1980).

La hidrogeología del valle está en estrecha dependencia con la geomorfología, ya que mientras el cordón medanoso central actúa como área de recarga, los salitrales, lagunas y bajos laterales lo hacen como sectores de descarga. El agua subterránea se aloja en la secuencia clástica, consistente en un manto arenoso, de hasta 15 metros de potencia, debajo del cual aparecen los limos arenosos del pampeano, que presentan intercalaciones de niveles arcillosos y calcáreos. El espesor total de los sedimentos se incrementa, hacia el este, de 150 a $250 \mathrm{~m}$, ya que, subyaciendo al pampeano, aparecen las formaciones geológicas propias de la cuenca de Macachín (Mariño et al., 2002). La secuencia acuífera muestra un efecto de drenaje diferido, con una transmisividad que normalmente varía entre 2 y $34 \mathrm{~m}^{2} /$ día y un coeficiente 




Figura 1. Ubicación del Valle Argentino.

Figure 1. Location of Valle Argentino.

de almacenamiento de $1 \times 10^{-3}$. Este último, es un valor aparente surgido de la interpretación de los ensayos de bombeo disponibles pero resultan esperables valores más elevados en el nivel arenoso superior.

La profundidad del nivel freático se sitúa entre 1 y $21 \mathrm{~m}$ en el cordón medanoso y un máximo de 100 $\mathrm{m}$ en la planicie. Los caudales que pueden extraerse alcanzan $25 \mathrm{~m}^{3} / \mathrm{h}$ en perforaciones de 150 metros de profundidad, aunque el uso de sistemas no convencionales (trincheras) permitió obtener hasta $90 \mathrm{~m}^{3} / \mathrm{h}$ (Schulz, 2004).

\section{Metodología}

\section{Balance hídrico del sistema}

En función de la información disponible, el balance del sistema hídrico se planteó para la localidad de General Acha y el paraje Padre Buodo. Para la resolución del balance diario en el suelo edáfico, la zona no saturada y el acuífero, se utilizó el código Visual Balan V. 2.0 (Samper et al., 2005). Para aplicar esta metodología, los distintos parámetros requeridos por el programa se tomaron de antecedentes o tablas provistas por el mismo programa, como se detalla en la tabla 1.
Los términos que pueden usarse para el balance son las entradas (precipitación y riego), las salidas (interceptación, escorrentía superficial, evapotranspiración, flujo hipodérmico y flujo subterráneo) y la variación del contenido de humedad del suelo y del nivel de agua en el acuífero (Samper et al., 1999). EI código Visual Balan sectoriza el medio subterráneo en tres componentes: a) el suelo edáfico, a través del cual infiltra el agua de lluvia o riego y tienen lugar los procesos de evaporación y transpiración, b) la zona no saturada, en la que pueden existir flujos tanto laterales (subhorizontales) como verticales y c) el acuífero o zona saturada, que recibe la recarga y puede tener flujos de descarga. El flujo de agua entre estos componentes se desarrolla a partir de la precipitación, una parte de la cual puede ser interceptada por la vegetación y el resto se distribuye entre escorrentía superficial e infiltración. Una parte del agua infiltrada vuelve a la atmósfera por evapotranspiración, otra parte se utiliza para aumentar la reserva de agua en el suelo y el resto constituye la recarga en tránsito. Este flujo es la entrada de agua a la zona no saturada, desde la cual puede fluir lateralmente a la atmósfera en forma de flujo hipodérmico o percolar verticalmente, constituyendo la recarga del acuífero si no hay descargas intermedias. La descarga subterránea es la salida natural hacia cuerpos de agua superficial o manantiales. El volumen de agua que Ilega a la zona saturada equivale a la recarga, la cual genera ascensos del nivel freático, que pueden ser contrastados con datos piezométricos ingresados por el usuario (Samper et al., 2005).

Cabe aclarar que Visual Balan plantea el modelo conceptual de una cuenca, donde la variación de niveles piezométricos (o caudales) se deben exclusivamente a la recarga dentro de la misma. Al aplicar el código a un sistema abierto, se introduce una incertidumbre en la evaluación de la recarga debida a la posible existencia de flujos laterales que influyan en la variación de los niveles. Se puede aceptar que, en este trabajo, dicha afectación se vería minimizada al haberse seleccionado puntos de control ubicados exclusivamente en la parte más elevada del cordón medanoso.

Análisis de las fluctuaciones del nivel freático

Este método (Healy y Cook, 2002) asume que el ascenso del nivel freático en acuíferos libres se debe exclusivamente al agua de recarga. Entonces, si se considera un período sin explotación (o puntos de control no influenciados por ella), es posible calcular la recarga de un acuífero en un área dada a partir de datos de fluctuación del nivel freático y de cómputos del caudal subterráneo entrante y saliente, de acuerdo con la siguiente ecuación:

$$
\mathrm{R}=(\Delta \mathrm{h} . \mathrm{S})-[((\mathrm{Qi}-\mathrm{Q} \mathrm{o}) / \mathrm{A}) \cdot \Delta \mathrm{t}]
$$

donde: $\mathrm{R}$ : recarga (L); $\Delta \mathrm{h}$ : Fluctuación del nivel freático en el período considerado (L), S: porosidad efectiva; Qi y Qo: Caudal subterráneo entrante y saliente respectivamente $\left(L^{3} . T^{-1}\right) ; A$ : Área del acuífero $\left(L^{2}\right)$ y $\Delta t$ : 


\begin{tabular}{|c|c|c|c|}
\hline & Parametro & General Acha & Padre Buodo \\
\hline \multirow{5}{*}{$\begin{array}{ll}0 & 0 \\
0 & 0 \\
0 \\
0 \\
0 \\
0 \\
0\end{array}$} & Período & $1981 / 2016$ & $1982 / 2016$ \\
\hline & Precipitación media anual $(\mathrm{mm})$ & 626,0 & 639,5 \\
\hline & ETP media anual (mm) & \multicolumn{2}{|c|}{$752,3(1976 / 95)$} \\
\hline & ETR & \multicolumn{2}{|c|}{ Método lineal- Valor umbral de déficit $(\mathrm{CBML})=100 \mathrm{~mm}$} \\
\hline & Recarga en tránsito & \multicolumn{2}{|c|}{ Cálculo por el método convencional sin flujo preferente } \\
\hline \multirow{6}{*}{$\begin{array}{l}\frac{0}{0} \\
\stackrel{0}{D}\end{array}$} & Porosidad total (\%) & \multicolumn{2}{|c|}{35 (Valor tabulado para suelos arenosos) } \\
\hline & Capacidad de campo $(\mathrm{mm})$ & \multicolumn{2}{|c|}{160 (Ídem anterior) } \\
\hline & Punto de marchitez (mm) & \multicolumn{2}{|c|}{60 (Ídem anterior) } \\
\hline & Espesor (m) & \multicolumn{2}{|c|}{1 (Valor adoptado según la vegetación predominante) } \\
\hline & Humedad inicial (mm) & \multicolumn{2}{|c|}{60 (Valor igual al punto de marchitez) } \\
\hline & Agua útil $(\mathrm{mm})$ & \multicolumn{2}{|c|}{100} \\
\hline \multirow{3}{*}{$\sum_{N}^{\infty}$} & Coef. agotamiento flujo hipodérmico $\left(d^{-1}\right)\left({ }^{*}\right)$ & 0,77 & 0,75 \\
\hline & Conductividad hidráulica vert. $(\mathrm{m} / \mathrm{d})\left({ }^{*}\right)$ & \multicolumn{2}{|c|}{0,5} \\
\hline & Ecuación de flujo & \multicolumn{2}{|c|}{ Resolución por el método explícito } \\
\hline \multirow{6}{*}{ 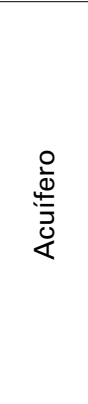 } & Discretización & \multicolumn{2}{|c|}{ Método de celda única } \\
\hline & Coef. agotamiento del acuífero $\left(\mathrm{d}^{-1}\right)\left({ }^{*}\right)$ & $\begin{array}{l}0,0007\left(\mathrm{PN}^{\circ} 9\right) \\
0,0009\left(\mathrm{PN}^{\circ} 8\right)\end{array}$ & 0,0007 \\
\hline & Coeficiente de almacenamiento $(-)(*)$ & $0,08\left(\mathrm{PN}^{\circ} 8\right)$ & $0,08\left(\mathrm{PN}^{\circ} 4\right)$ \\
\hline & \begin{tabular}{|l} 
Puntos de control piezométrico \\
\end{tabular} & $\frac{0,09\left(\mathrm{PN}^{\circ} 9\right)}{2\left(\mathrm{PN}^{\circ} 8 \text { y } \mathrm{PN}^{\circ} 9\right)}$ & $\frac{0,09\left(\mathrm{PN}^{\circ} 3\right)}{2\left(\mathrm{PN}^{\circ} 3 \text { y } \mathrm{PN}^{\circ} 4\right)}$ \\
\hline & Nivel inicial (msnm) & $\begin{array}{l}216,79\left(\mathrm{PN}^{\circ} 8\right) \\
211,81\left(\mathrm{PN}^{\circ} 9\right)\end{array}$ & $\begin{array}{l}177,88\left(\mathrm{PN}^{\circ} 3\right) \\
163,04\left(\mathrm{PN}^{\circ} 4\right)\end{array}$ \\
\hline & Nivel de referencia (msnm) & $215,61\left(\mathrm{PN}^{\circ} 8\right)$ & $177,55\left(\mathrm{PN}^{\circ} 3\right)$ \\
\hline
\end{tabular}

Tabla 1. Parámetros utilizados en el programa Visual Balan (* valores ajustados).

Table 1. Parameters applied to the Visual Balan code (* adjusted values).

\section{Período de aplicación del balance.}

Si se considera un período sin recarga, la fluctuación del nivel freático es igual a la diferencia entre el caudal subterráneo entrante y saliente del área analizada. Si durante tales intervalos los niveles se profundizan, la lámina de descenso equivale a la que sale del área por escurrimiento subterráneo. Esta fluctuación negativa es asimilable a lo que Olin (1995) denomina «tasa de recesión», la cual puede obtenerse a partir del descenso del nivel freático correspondiente a períodos sin precipitación.

Las estimaciones de la recarga, para el período 1996-2016, se basaron en los registros freatimétricos que la Administración Provincial del Agua toma periódicamente en dos líneas de puntos de control. Un grupo de freatímetros, que incluye del número 1 al 5, están emplazados sobre la ruta Nac. 35, en cercanías del paraje Padre Buodo. El segundo grupo, ubicado sobre la Ruta Prov. 9, desde General Acha a Utracán, incluye los freatímetros 6 al 12 (Figura 2). Para este trabajo se consideraron, en la zona de Padre Buodo, los pozos 2 (con datos sólo hasta 2005), 3 (con datos sólo hasta 2002) y 4, mientras que en General Acha se tuvieron en cuenta los puntos 7, 8 y 9. La serie de registros se dividió en subperíodos de duración aproximadamente anual, entre junio de un año y mayo del siguiente, y para cada uno se calculó la fluctuación media del conjunto de pozos considerados.

\section{Resultados}

\section{Balance hídrico del sistema}

Los resultados medios anuales del balance hídrico para los dos sitios evaluados se resumen en la tabla 2.

\begin{tabular}{|l|c|c|}
\hline Localidad & Padre Buodo & General Acha \\
Recarga media anual & 39,5 & 35,3 \\
Rango de la recarga & 0 a 81 & 0 a 76 \\
Evapotranspiración real & 484,3 & 472,8 \\
Error cuadrático medio & $0,542-0,583$ & $0,363-0,391$ \\
\hline
\end{tabular}

Tabla 2. Resultados del balance hídrico para los sitios evaluados. Table 2. Results of water balance for the evaluated sites.

Las figuras 3 y 4 muestran el ajuste entre los niveles freáticos calculados y medidos para un punto de control de cada sitio, donde se observa una concordancia aceptable, aunque con errores cuadráticos medios algo mayores en el caso de Padre Buodo.

Los freatigramas muestran que los niveles más someros ocurren en 2001 y 2002, coincidentemente con tres períodos consecutivos cuya recarga anual promedio para ambos sitios fue de $67,7 \mathrm{~mm}$ (mínima de $53 \mathrm{~mm}$ y máxima de $81 \mathrm{~mm}$ ), asociada a precipitaciones superiores a $800 \mathrm{~mm} / \mathrm{año}$. Luego se mantiene una tendencia gradualmente descendente, que llega a la mayor profundización entre 2013 y 2014, cuando el nivel freático 


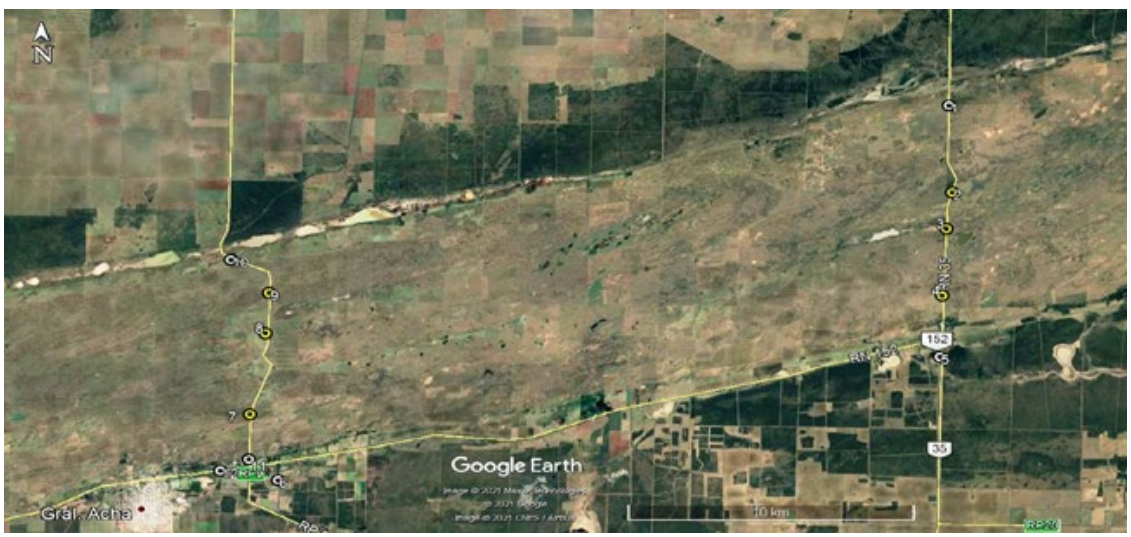

Figura 2. Imagen del sector estudiado con la ubicación de los piezómetros.

Figure 2. Study area, showing locations of the observation wells.

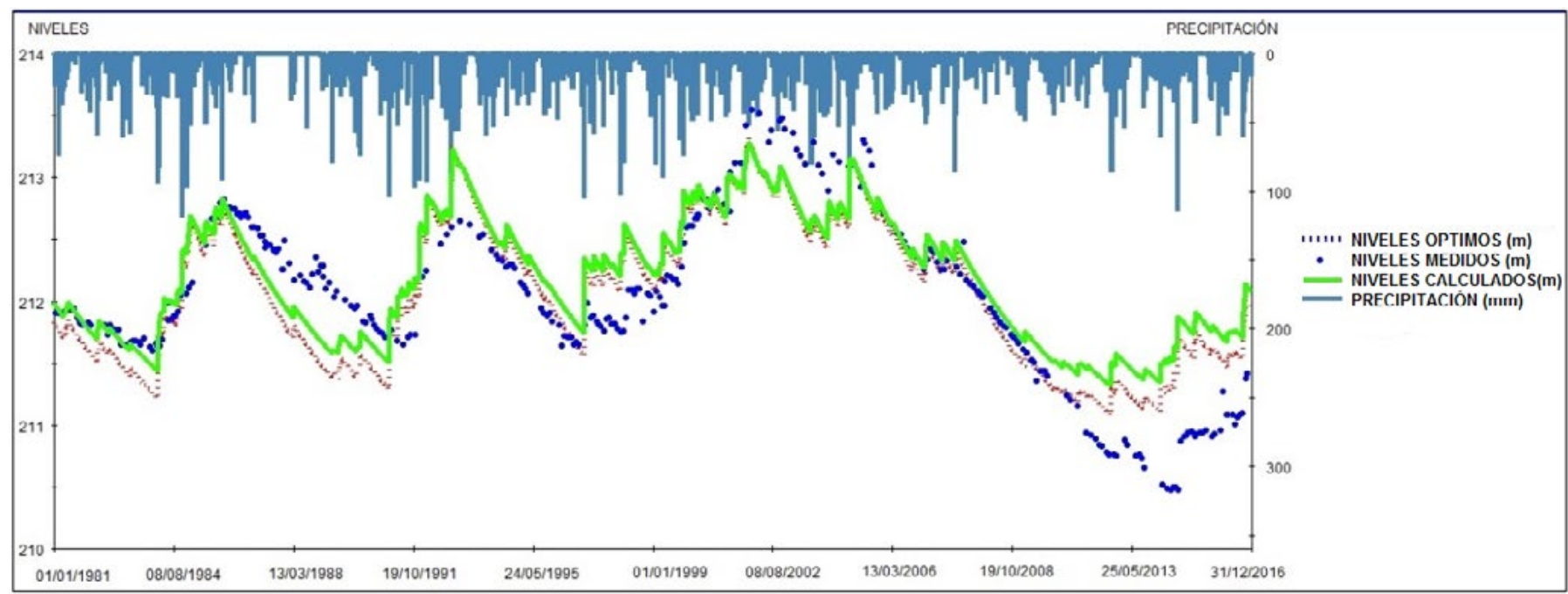

Figura 3. Ajuste de niveles (en $\mathrm{m}$ snm) calculados y medidos para el piezómetro $\mathrm{N}^{\circ} 9$ (General Acha).

Figure 3. Adjustment between calculated and measured water levels (in $\mathrm{m}$ snm) for observation well $N^{\circ} 9$ (General Acha).

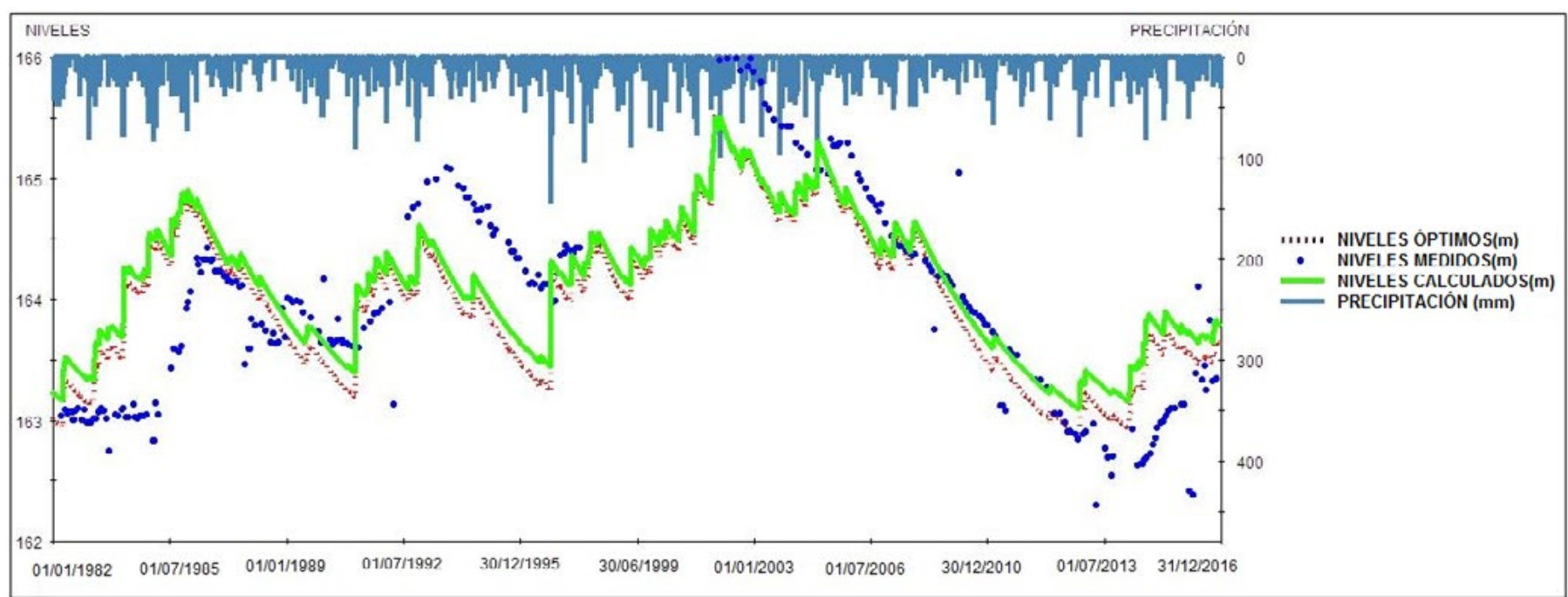

Figura 4. Ajuste de niveles (en msnm) calculados y medidos para el piezómetro $\mathrm{N}^{\circ} 4$ (Padre Buodo).

Figure 4. Adjustment between calculated and measured water levels for observation well N4 (Padre Buodo). 
cae entre 3 y 3,70 metros con respecto a su posición más elevada. Este último estadio es el resultado de 4 períodos previos y consecutivos con precipitaciones inferiores a $500 \mathrm{~mm}$, dando lugar a una recarga promedio de 5,2 $\mathrm{mm}$ (mínima de 0 y máxima de $12 \mathrm{~mm}$ ).

\section{Análisis de las fluctuaciones del nivel freático}

La tabla 3 muestra una síntesis de la precipitación y la fluctuación media del nivel freático en los distintos subperíodos para Padre Buodo y General Acha.

La recarga promedio anual para los 20 subperíodos calculados fue de 46,6 mm para General Acha, con un rango entre 21 y $91 \mathrm{~mm}$, y de $53,4 \mathrm{~mm}$ para Padre Buodo (mínimo de 0 y máximo de $112 \mathrm{~mm}$ ). En ambos casos se adoptó una porosidad efectiva de 0,07 , que es el menor de los valores ajustados por el método anterior, y la tasa de recesión aplicada fue de $-0,13 \mathrm{~mm} / \mathrm{d}$, que se obtuvo de los registros correspondientes a General Acha y como promedio de 7 intervalos con precipitación nula o inferior a $3 \mathrm{~mm}$. El valor resultante para $\mathrm{Pa}$ dre Buodo $(-0,38 \mathrm{~mm} / \mathrm{d})$ se descartó, ya que producía sobreestimaciones significativas de la recarga.

En Padre Buodo se destacan dos lapsos que registraron precipitaciones superiores a $800 \mathrm{~mm}$ (de junio 1996 a junio 1998 y de junio 1999 a junio 2002), cuando se produjeron ascensos del nivel freático de 0,89 y 1,02 metros, respectivamente. En estos subperíodos se estimaron valores de recarga que variaron entre 50 y $96 \mathrm{~mm}$. Por el contrario, en el subperíodo que va de junio 2009 a junio 2010, que acumuló una precipitación de $434 \mathrm{~mm}$ y fue precedido por otro con sólo 382 $\mathrm{mm}$, tuvo lugar un descenso $0,82 \mathrm{~m}$, asociado a valores nulos de recarga.

El mismo escenario se verifica para General Acha durante los dos períodos húmedos, aunque con precipitaciones anuales iguales o superiores a $740 \mathrm{~mm}$.

\begin{tabular}{|c|c|c|c|c|}
\hline \multirow{2}{*}{ Fecha } & \multicolumn{2}{|c|}{ Padre Buodo } & \multicolumn{2}{c|}{ General Acha } \\
\cline { 2 - 5 } & $\mathbf{P}$ & $\Delta$ NF & $\mathbf{P}$ & $\Delta$ NF \\
\hline $07 / 06 / 1996$ & & & & \\
\hline $03 / 06 / 1997$ & 802,0 & 0,40 & 741,3 & 0,15 \\
\hline $12 / 06 / 1998$ & 931,5 & 0,49 & 808,0 & 0,45 \\
\hline $01 / 06 / 1999$ & 690,5 & 0,03 & 724,0 & $-0,01$ \\
\hline $10 / 05 / 2000$ & 807,1 & 0,37 & 896,0 & 0,46 \\
\hline $21 / 06 / 2001$ & 801,5 & 0,61 & 759,5 & 0,54 \\
\hline $18 / 06 / 2002$ & 871,0 & 0,04 & 740,0 & 0,10 \\
\hline $09 / 06 / 2003$ & 645,5 & $-0,42$ & 596,0 & $-0,34$ \\
\hline $01 / 06 / 2004$ & 753,5 & $-0,32$ & 721,0 & 0,15 \\
\hline $14 / 05 / 2005$ & 693,0 & 0,02 & 772,5 & $-0,34$ \\
\hline $16 / 06 / 2006$ & 571,5 & $-0,43$ & 557,5 & $-0,32$ \\
\hline $31 / 05 / 2007$ & 729,0 & $-0,40$ & 726,5 & $-0,06$ \\
\hline $28 / 05 / 2008$ & 535,5 & $-0,69$ & 602,0 & $-0,27$ \\
\hline $05 / 06 / 2009$ & 382,0 & 0,19 & 349,0 & $-0,38$ \\
\hline $18 / 06 / 2010$ & 434,0 & $-0,82$ & 491,0 & $-0,40$ \\
\hline $03 / 06 / 2011$ & 462,0 & 0,22 & 611,0 & $-0,04$ \\
\hline $22 / 06 / 2012$ & 441,0 & $-0,43$ & 468,0 & $-0,37$ \\
\hline $04 / 07 / 2013$ & 610,0 & $-0,14$ & 611,5 & 0,00 \\
\hline $18 / 06 / 2014$ & 580,0 & $-0,14$ & 541,5 & $-0,12$ \\
\hline $02 / 06 / 2015$ & 645,5 & 0,45 & 622,5 & 0,42 \\
\hline $06 / 06 / 2016$ & 620,0 & 0,26 & 686,5 & 0,16 \\
\hline
\end{tabular}

Tabla 3. Precipitación ( $P$ en $\mathrm{mm}$ ) y fluctuación media del nivel freático $(\triangle N F$ en $m)$ en los sitios evaluados.

Table 3. Rainfall ( $P$ in $\mathrm{mm}$ ) and mean water table fluctuation ( $\triangle N F$ in $m)$ for the evaluated sites.
Considerando la fluctuación media de los tres puntos de control utilizados, los ascensos del nivel freático fueron de 0,60 y 1,10 metros, respectivamente, vinculados a recargas anuales estimadas entre 54 y $91 \mathrm{~mm}$. En el lapso más seco, con precipitaciones similares al sitio anterior y una recarga de sólo $21 \mathrm{~mm}$, se produjo un descenso de 0,40 metros.

\section{Conclusiones}

La integración de los resultados presentados indica que, bajo condiciones naturales, en períodos con precipitaciones anuales superiores a $740 \mathrm{~mm}$, potencialmente generadoras de recargas anuales mayores a 50 $\mathrm{mm}$, son esperables ascensos del nivel freático entre 0,60 y poco más de 1 metro. Por el contrario, cuando la recarga es nula o exigua (menor a $20 \mathrm{~mm} / a n ̃ o)$, asociada a lluvias inferiores a $500 \mathrm{~mm} /$ año, ocurrirían descensos de 0,40 a 0,80 metros, debidos al predominio de la descarga subterránea. En este contexto, los valores medios de recarga obtenidos del modelo Visual Balan representarían un escenario de equilibrio o con fluctuaciones leves del nivel freático.

Este modelo conceptual es una simplificación de la dinámica del sistema, dado que la respuesta del nivel freático no es lineal respecto a las precipitaciones del período considerado, sino que también incide su repartición estacional y la pluviometría de los períodos antecedentes.

No obstante, la variabilidad descripta, que es característica de las regiones semiáridas, da cuenta de la sensibilidad del sistema hídrico ante las variaciones pluviométricas $y$, consecuentemente, de la recarga y alerta sobre la importancia de una correcta gestión del agua subterránea. Tales acciones deberán orientarse a evitar una eventual sobrexplotación y así lograr la preservación cuantitativa y cualitativa del recurso. En tal sentido, se recomienda intensificar los controles freatimétricos e hidroquímicos a fin de mejorar el modelo hidrogeológico conceptual. Además, por tratarse de un medio natural altamente demandante de soluciones sistémicas, se debería fomentar la conformación de grupos de trabajo en gestión de acuíferos que, en función de aspectos metodológicos definidos, orienten la definición y puesta en práctica de un Plan de Ordenación Territorial. Dicha planificación tendrá una componente social y económica significativa, ya que deberá contemplar y armonizar la demanda, actual y futura, para el consumo humano de las localidades abastecidas por el acuífero con los requerimientos de otros usuarios, como son el sector rural e industrial.

\section{Agradecimientos}

La Administración Provincial del Agua suministró los registros freatimétricos y datos de precipitaciones diarias que sirvieron de base para este trabajo.

El Dr. Emilio Custodio aportó comentarios y sugerencias que contribuyeron a una mejora notable del artículo. 


\section{Referencias}

Cano, E. G.; Casagrande, H. A.; Conti, B.; Fernández, R.; Hevia, J. C.; Lea Plaza, D.; Maldonado Pinedo, H.; Martínez, M. A.; Montes y Peña Zubiate, C. A., 1980. Inventario Integrado de los Recursos Naturales de la Provincia de La Pampa. I.N.T.A, Provincia de La Pampa y UNLPam, Santa Rosa, 493 pp.

Castro, E. y Tullio J.O., 1990. Acuífero Valle Argentino (Síntesis Preliminar). Informe inédito. Administración Provincial del Agua, Santa Rosa.

Cavalié, C., 1985. Estudio Hidrogeológico Hoja General Acha. Revista Pampa Geológica, Publicación Especial, 64-80.

Dornes, P. y Schulz, C., 2001. Determinación de la recarga en la región de La Pampa central, Argentina. En: Medina, A., Carrera, J. y Vives, L. (eds.), Las caras del agua subterránea. Serie Hidrogeología y Aguas Subterráneas, Instituto Tecnológico Geominero, Madrid, 1(I), 614-622.

Galea, J.M., 2017. Caracterización Hidrogeológica del Valle Argentino, Sector General Acha-El Carancho. Tesis de grado inédita. Facultad de Ciencias Exactas y Naturales, Universidad Nacional de La Pampa, 63 pp.

Healy, R.W. and Cook, P.G., 2002. Using groundwater levels to estimate recharge. Hydrogeology Journal, 10, 91-109.

Mariño, E.E. y Schulz, C.J., 2008. Importancia de los acuíferos en ambiente medanoso en la región semiárida pampeana. Huellas, 12, 113-127.

Mariño E.E., Schulz, C.J., Fernandez, M.A., Castro, E. y Dalmaso, M.G., 2002. Hidroquímica subterránea del sector oriental del Valle Argentino, provincia de La Pampa. VIII Jornadas Pampeanas de Ciencias Naturales, Santa Rosa, 139-141.

Martínez, H. y Fiorucci, E., 1998. Planificación Regional Integral-Estudio de Suelos del Valle Argentino. Informe inédito. Subsecretaría de Asuntos Agrarios, Santa Rosa.

Olin, M., 1995. Estimation of base level for an aquifer from recession rates of groundwater levels. Hydro- geology Journal, 3(2), 40-51.

Samper, J., Huguet, LI., Ares, J. y García-Vera, M.A., 1999. Modelos interactivos de balance hidrológico. En: Muñoz-Carpena, R., Ritter, A. yTascón, C. (eds.), Estudios de la Zona No Saturada del Suelo, ICIA, Tenerife, 187-193.

Samper, J., Huguet, L., Ares, J. y García-Vera, M.A. 2005. Manual del usuario del programa VISUAL BALAN V. 2.0. ENRESA, Madrid, 139 pp.

Schulz, C.J., 2004. Estudio hidrogeológico del area central del valle argentino, La Pampa, Argentina. Elaboración de una propuesta de gestión de los recursos hídricos. Tesis doctoral inédita. Universidad Nacional de Córdoba, $141 \mathrm{pp}$.

Schulz, C., Bonorino, G., Vives, L. y Dornes, P., 1997. Estudio de Planificación y Gestión de los Recursos Hídricos del Valle Argentino, Provincia de La Pampa. Congreso Nacional de Hidrogeología, Bahía Blanca, 403-414.

Schulz, C.J., Dornes, P. F., Vives, L.S. y Bonorino, A.G., 1998. Caracterización hidrogeológica del acuífero detrítico del Valle Argentino, La Pampa, Argentina, con énfasis en el estudio de la recarga. $4^{\circ}$ Congreso Latinoamericano de Hidrología Subterránea, Montevideo, 3, 1457-1467.

Schulz, C.J., Mariño, E.E., Castro, E., Dalmaso, M.G., Fernández, A. y Dornes, P., 1999. Evaluación preliminar de la calidad del agua subterránea en un sector del Valle Argentino, provincia de La Pampa. VII Jornadas Pampeanas de Ciencias Naturales, Santa Rosa, 289-296.

Schulz C. J., Castro, E.C. y Dalmaso G., 2002. Hidrogeoquímica del Acuífero del Valle Argentino (L.P). Aptitud del Agua Para Uso Humano y Rural. XXXII IAH \& VIALHSUD Congress, Mar del Plata, 277-286.

Universidad Ben Gurion del Negev, 1998. Valle Argentino. Planificación Regional Integral. Informe inédito, Parte I, 151pp.

Universidad Nacional de La Pampa (UNLPam), 2015. Inventario de los Recursos Hídricos de la Provincia de La Pampa. Informe inédito (Tomo I), Secretaría de Recursos Hídricos de La Pampa, 168 pp.
Recibido: julio 2019

Revisado: noviembre 2019

Aceptado: enero 2020

Publicado: marzo 2021 
